Research Article

Open Access

Mamoru Nunokawa, Janusz Sokót*, and Huo Tang

\title{
Length problems for Bazilevič functions
}

https://doi.org/10.1515/dema-2019-0007

Received October 4, 2018; accepted December 30, 2018

Abstract: Let $C(r)$ denote the curve which is image of the circle $|z|=r<1$ under the mapping $f$. Let $L(r)$ be the length of $C(r)$ and $A(r)$ the area enclosed by the curve $C(r)$. Furthermore $M(r)=\max _{|z|=r}|f(z)|$. We present some relations between these notions for Bazilevič functions.

Keywords: Bazilevič function, close-to-convex functions, convex functions, starlike function, convex function

MSC: 30C45, 30C80

\section{Preliminaries}

Let $\mathcal{H}$ denote the class of functions $f$ which are analytic in the unit disk $\mathbb{D}=\{z \in \mathbb{C}:|z|<1\}$, and $\mathcal{A}$ be the subclass of $\mathcal{H}$ consisting of functions normalized by $f(0)=0=f^{\prime}(0)-1$. Let $\mathcal{S} \subset \mathcal{A}$ be the class of functions univalent (i.e. one-to-one) in $\mathbb{D}$. Denote by $\mathcal{S}^{*}$ the subclass of $\mathcal{S}$ of starlike functions, i.e. the class of functions $f \in \mathcal{A}$ such that $f(\mathbb{D})$ is starlike with respect to the origin. It is well-known, since the work of [1], that $f \in \mathcal{S}^{*}$ if, and only if, $f \in \mathcal{A}$ and

$$
\mathfrak{R e}\left\{\frac{z f^{\prime}(z)}{f(z)}\right\}>0, z \in \mathbb{D} .
$$

Recall that a set $E \subset \mathbb{C}$ is said to be starlike with respect to to the origin if, and only if, the linear segment joining 0 to every other point $w \in E$ lies entirely in $E$. By $\mathcal{P}$ we denote the class of Carathéodory functions $p$ which are analytic in $\mathbb{D}$, satisfying the condition $\mathfrak{R e}\{p(z)\}>0$ for $z \in \mathbb{D}$, with $p(0)=1$.

Suppose now that $f \in \mathcal{A}$, then $f$ is close-to-convex if, and only if, there exists $\alpha \in(-\pi / 2, \pi / 2)$, and a function $g \in \mathcal{S}^{\star}$ such that

$$
\mathfrak{R e}\left\{e^{i \alpha} \frac{z f^{\prime}(z)}{g(z)}\right\}>0, z \in \mathbb{D} .
$$

This class of close-to-convex functions was introduced in [2]. Functions defined by (1.1) with $\alpha=0$ were considered earlier by Ozaki [3], see also Umezawa [4, 5]. Moreover, Lewandowski [6, 7] defined the class of functions $f \in \mathcal{A}$ for which the complement of $f(\mathbb{U})$ with respect to the complex plane is a linearly accessible domain in the large sense. The Lewandowski class is identical with the class of close-to-convex functions. Here, we denote this class by $\mathcal{K}$, and note that $\mathcal{S}^{\star} \subset \mathcal{K} \subset \mathcal{S}$. The class of close-to-convex functions forms an important subclass of $\mathcal{S}$. Length problems for close-to-convex functions were recently considered in [8]. A proper subset of $\mathcal{K}$ is the class of bounded boundary rotation of $f$ such that $f^{\prime}(z) \neq 0$ in the unit disc and

$$
4 \pi \leq \lim _{r \rightarrow 1} \int_{0}^{2 \pi}\left|\mathfrak{R e}\left\{1+\frac{z f^{\prime \prime}(z)}{f^{\prime}(z)}\right\}\right| \mathrm{d} \theta, \quad z=r e^{i \theta} .
$$

Mamoru Nunokawa: University of Gunma, Hoshikuki-cho 798-8, Chuou-Ward, Chiba, 260-0808, Japan; E-mail: mamoru_nuno@doctor.nifty.jp

*Corresponding Author: Janusz Sokół: University of Rzeszów, Faculty of Mathematics and Natural Sciences, ul. Prof. Pigonia 1, 35-310 Rzeszów, Poland; E-mail: jsokol@ur.edu.pl

Huo Tang: School of Mathematics and Statistics, Chifeng University, Chifeng 024000, Inner Mongolia, China;

E-mail: thth2009@163.com 
Another even larger subset of $\mathcal{S}$ is formed by the Bazilevič functions. Bazilevič [9] introduced a class of functions $f \in \mathcal{A}$ which are defined by the following

$$
f(z)=\left\{\frac{\beta}{1+\alpha^{2}} \int_{0}^{z}(h(z)-i \alpha) \zeta^{\left(-\alpha \beta /\left(1+\alpha^{2}\right)\right)-1} g^{\beta /\left(1+\alpha^{2}\right)}(\zeta) \mathrm{d} \zeta\right\}^{(1+i \alpha) / \beta},
$$

where $h \in \mathcal{P}$ and $g \in \mathcal{S}^{*}, \alpha$ is any real number and $\beta>0$. Bazilevič showed that all such functions are univalent in $\mathbb{D}$. Putting $\alpha=0$ in (1) and differentiating it, we have

$$
z f^{\prime}(z)=(f(z))^{1-\beta}(g(z))^{\beta} h(z)
$$

and

$$
\mathfrak{R e}\{h(z)\}=\mathfrak{R e}\left\{\frac{z f^{\prime}(z)}{f^{1-\beta}(z) g^{\beta}(z)}\right\}>0, \quad z \in \mathbb{D} .
$$

Thomas [10] called a function satisfying condition (1.2) a Bazilevič function of type $\beta$. For further works on Bazilevič functions we refer to [11]-[15]. It is easy to see that Bazilevič functions of type $\beta=1$ are close-toconvex functions, univalent in $\mathbb{D}$. Furthermore, the set of starlike functions is contained in the set of Bazilevič functions of type $\beta$.

Let $C(r)$ denote the curve which is image of the circle $|z|=r<1$ under the mapping $f$. Let $L(r)$ be the length of $C(r)$ and $A(r)$ the area enclosed by the curve $C(r)$. Furthermore $M(r)=\max _{|z|=r}|f(z)|$. In [16], Thomas has shown the following:

Theorem 1.1. [16, Th.1] If $g \in \mathcal{S}^{\star}$, then

$$
L(r) \leq 2 \sqrt{\pi A(r)}\left(1+\log \frac{1+r}{1-r}\right) \quad \text { as } \quad r \rightarrow 1 .
$$

Note that in [17], Thomas considered $L(r)$ for the class of bounded close-to-convex functions and asked the following question.

Does there exist a starlike function for which

$$
\liminf _{r \rightarrow 1} \frac{L(r)}{M(r) \log \frac{1}{1-r}}>0
$$

or

$$
\liminf _{r \rightarrow 1} \frac{L(r)}{\sqrt{A(r)} \log \frac{1}{1-r}}>0 ?
$$

Applying the result of [18], we give a negative partial result of the above open problem (1.3). Some related problems were considered in $[19,20]$.

\section{On Bazilevič functions of bounded rotation}

The following lemma is due to Pommerenke [21].

Lemma 2.1. [21] Let $f(z)=z+\sum_{n=2}^{\infty} a_{n} z^{n}$ be analytic and univalent in $\mathbb{D}$. Then we have

$$
M(r)=\frac{4}{\sqrt{\pi}}\left(A(r) \log \frac{3}{1-r}\right)^{1 / 2} \text { as } r \rightarrow 1 .
$$

Lemma 2.2. Let $f(z)=z+\sum_{n=2}^{\infty} a_{n} z^{n}$ be analytic in $\mathbb{D}$. Then we have

$$
M(r) \leq \mathcal{O}\left(S(\sqrt{r}) \log \frac{1}{1-r}\right)^{1 / 2} \text { as } r \rightarrow 1,
$$


where $\mathcal{O}$ means the Landau's symbol and

$$
S(r)=\frac{1}{2 \pi} \int_{0}^{r} \int_{0}^{2 \pi} \rho\left|f^{\prime}\left(\rho e^{i \theta}\right)\right|^{2} \mathrm{~d} \theta \mathrm{d} \rho .
$$

Proof. Assume that $0<r_{1}<r, \zeta=\sqrt{\rho} e^{i \varphi}, 0 \leq|t| \leq r, 0<\rho<r$ and throughout $C$ will denote an absolute constant not necessarily the same each time. We have

$$
|f(z)|=\left|\int_{0}^{z} f^{\prime}(t) \mathrm{d} t\right|
$$

Now, by using the substitution

$$
t=\rho e^{i \theta}, \mathrm{d} t=e^{i \theta} \mathrm{d} \rho, \zeta=\sqrt{\rho} e^{i \varphi},
$$

this becomes

$$
\begin{aligned}
|f(z)| & =\left|\int_{0}^{r} f^{\prime}\left(\rho e^{i \theta}\right) e^{i \theta} \mathrm{d} \rho\right| \\
& \leq \int_{0}^{r}\left|f^{\prime}\left(\rho e^{i \theta}\right)\right| \mathrm{d} \rho \\
& \leq \frac{1}{2 \pi} \int_{0}^{r} \int_{|\zeta|=\sqrt{\rho}} \frac{\left|f^{\prime}(\zeta)\right|}{\left|\zeta-\rho e^{i \theta}\right|}|\mathrm{d} \zeta| \mathrm{d} \rho \\
& \leq \frac{1}{2 \pi} \int_{0}^{r_{1}} \int_{0}^{2 \pi} \sqrt{\rho} \frac{\left|f^{\prime}(\zeta)\right|}{\left|\zeta-\rho e^{i \theta}\right|} \mathrm{d} \varphi \mathrm{d} \rho+\frac{1}{2 \pi} \int_{r_{1}}^{r} \int_{0}^{2 \pi} \sqrt{\frac{\rho}{r_{1}}} \frac{\left|f^{\prime}(\zeta)\right|}{\left|\zeta-\rho e^{i \theta}\right|} \mathrm{d} \varphi \mathrm{d} \rho \\
& \leq C+\frac{1}{2 \pi \sqrt{r_{1}}} \int_{r_{1}}^{r} \int_{0}^{2 \pi} \sqrt{\rho} \frac{\left|f^{\prime}(\zeta)\right|}{\mid \zeta-\rho e^{i \theta \mid}} \mathrm{d} \varphi \mathrm{d} \rho .
\end{aligned}
$$

Further, because

$$
\left(\iint_{D}|f(x, y) g(x, y)| \mathrm{d} x \mathrm{~d} y\right)^{2} \leq\left(\iint_{D}|f(x, y)|^{2} \mathrm{~d} x \mathrm{~d} y\right)\left(\iint_{D}|g(x, y)|^{2} \mathrm{~d} x \mathrm{~d} y\right),
$$

we have

$$
\begin{aligned}
& C+\frac{1}{2 \pi \sqrt{r_{1}}} \int_{r_{1}}^{r} \int_{0}^{2 \pi} \sqrt{\rho} \frac{\left|f^{\prime}(\zeta)\right|}{\mid \zeta-\rho e^{i \theta \mid}} \mathrm{d} \varphi \mathrm{d} \rho \\
\leq & C+\frac{1}{\sqrt{r_{1}}}\left(\frac{1}{2 \pi} \int_{r_{1}}^{r} \int_{0}^{2 \pi} \sqrt{\rho}\left|f^{\prime}\left(\sqrt{\rho} e^{i \varphi}\right)\right|^{2} \mathrm{~d} \varphi \mathrm{d} \rho\right)^{1 / 2}\left(\frac{1}{2 \pi} \int_{r_{1}}^{r} \int_{0}^{2 \pi} \frac{\sqrt{\rho}}{\left.\mid \sqrt{\rho} e^{i \varphi}-\rho e^{\left.i \theta\right|^{2}} \mathrm{~d} \varphi \mathrm{d} \rho\right)^{1 / 2}}\right. \\
= & C+\frac{1}{\sqrt{r_{1}}}\left(\frac{1}{2 \pi} \int_{0}^{r} \int_{0}^{2 \pi} \sqrt{\rho}\left|f^{\prime}\left(\sqrt{\rho} e^{i \varphi}\right)\right|^{2} \mathrm{~d} \varphi \mathrm{d} \rho\right)^{1 / 2}\left(\frac{1}{2 \pi} \int_{r_{1}}^{r} \frac{\sqrt{\rho}}{\rho-\rho^{2}} \mathrm{~d} \rho\right)^{1 / 2} \\
\leq & C+\frac{1}{\sqrt{r_{1}}}\left(\frac{1}{2 \pi} \int_{0}^{\sqrt{r}} \int_{0}^{2 \pi} \sqrt{\rho}\left|f^{\prime}\left(\sqrt{\rho} e^{i \varphi}\right)\right|^{2} \mathrm{~d} \varphi \mathrm{d} \rho\right)^{1 / 2}\left(\frac{1}{2 \pi \sqrt{r_{1}}} \int_{r_{1}}^{r} \frac{1}{1-\rho} \mathrm{d} \rho\right)^{1 / 2}
\end{aligned}
$$




$$
\begin{aligned}
& \leq C+\frac{1}{\sqrt{r_{1}}} \sqrt{S(\sqrt{r})}\left(\frac{1}{2 \pi \sqrt{r_{1}}} \int_{0}^{r} \frac{1}{1-\rho} \mathrm{d} \rho\right)^{1 / 2} \\
& =\mathcal{O}\left\{\sqrt{S(\sqrt{r})} \sqrt{\log \frac{1}{1-r}}\right\} \text { as } r \rightarrow 1,
\end{aligned}
$$

where $0<r_{1}<r<1$. Because $M(r)=\max _{|z|=r}|f(z)|$, we finally obtain (2.1).

Remark 1. If $f(z)=z+\sum_{n=2}^{\infty} a_{n} z^{n}$ is analytic and univalent in $\mathbb{D}$, then it is trivial that

$$
S(r)=A(r) \text { for } 0<r<1
$$

so in this case (2.1) becomes

$$
M(r) \leq \mathcal{O}\left(A(\sqrt{r}) \log \frac{1}{1-r}\right)^{1 / 2} \text { as } r \rightarrow 1 .
$$

Theorem 2.3. Let $f$ be a Bazilevič function of type $\beta$ and let $f$ be a function of bounded rotation on $0<|z|=$ $r<1$, and suppose that

$$
M(r)=\mathcal{O}\left\{(1-r)^{-\alpha} p(r)\right\} \text { as } r \rightarrow 1
$$

for all $\alpha$, where $0<\alpha \leq 2$, while $p(r)$ is monotone increasing function of $r$ in a wider sense, and $\mathcal{O}$ in (2.2) cannot be replaced by 0 . We then have

$$
L(r)=\mathcal{O}\left(A(r) \log \frac{1}{1-r}\right)^{1 / 2} \text { as } r \rightarrow 1
$$

Proof. From (2.2) and applying the same method as in the proof of Theorem 1 in [22], we have

$$
\begin{aligned}
L(r)= & \int_{0}^{2 \pi}\left|z f^{\prime}(z)\right| \mathrm{d} \theta \\
= & \int_{0}^{2 \pi}\left|(f(z))^{(1-\beta)}(g(z))^{\beta} h(z)\right| \mathrm{d} \theta \\
\leq & \int_{0}^{r} \int_{0}^{2 \pi}\left|(1-\beta) f^{\prime}(z) f^{-\beta}(g(z))^{\beta} h(z)\right| \mathrm{d} \theta \mathrm{d} \rho \\
& +\int_{0}^{r} \int_{0}^{2 \pi}\left|f^{1-\beta} \beta\left(g^{\prime}(z)\right)^{\beta-1} h(z)\right| \mathrm{d} \theta \mathrm{d} \rho+\int_{0}^{r} \int_{0}^{2 \pi}\left|f^{1-\beta}(g(z))^{\beta} h^{\prime}(z)\right| \mathrm{d} \theta \mathrm{d} \rho \\
= & I_{1}+I_{2}+I_{3}, \text { say. }
\end{aligned}
$$

Then, from [18, p.338], and from Lemma 2.1, we have the following

$$
I_{1} \leq 2 \pi|1-\beta| M(r)=\mathcal{O}\left(A(r) \log \frac{1}{1-r}\right)^{1 / 2} \text { as } r \rightarrow 1 .
$$

Next, we have (2.3) below from [22, p.277] and Lemma 2.1:

$$
\begin{aligned}
I_{2} & =C \int_{\delta}^{r} \frac{M(\rho)}{1-\rho} \mathrm{d} \rho+C \\
& \leq C \int_{\delta}^{r} \frac{p(\rho)}{(1-\rho)^{1+\alpha}} \mathrm{d} \rho+C \\
& \leq \frac{C p(r)}{\alpha}(1-r)^{-\alpha}+C
\end{aligned}
$$




$$
=\mathcal{O}(M(r))=\mathcal{O}\left(A(r) \log \frac{1}{1-r}\right)^{1 / 2} \text { as } r \rightarrow 1,
$$

where $\delta$ is fixed $0<\delta<\rho \leq r<1$. Applying the result of [22, p.277] and the same method as in the calculation (2.3), we have

$$
\begin{aligned}
I_{3} & =2 \pi\{|1-\beta| C+|\beta|\} \int_{0}^{r} \frac{M(\rho)}{1-\rho} \mathrm{d} \rho \\
& =\mathcal{O}\left(A(r) \log \frac{1}{1-r}\right)^{1 / 2} \text { as } r \rightarrow 1 .
\end{aligned}
$$

This completes the proof of Theorem 2.3.

From Theorem 2.3, we easily have the following corollary.

Corollary 2.4. Let $f$ be a Bazilevič function of type $\beta$ and let $f$ be a function of bounded rotation on $0<|z|=$ $r<1$ and suppose that

$$
M(r)=\mathcal{O}\left\{(1-r)^{-\alpha}\left(\log \frac{1}{1-r}\right)^{1 / 2}\right\} \text { as } r \rightarrow 1 .
$$

Then there is no Bazilevič function of type $\beta$ satisfying the condition (1.3).

\section{References}

[1] Study E., Konforme Abbildung Einfachzusammenhangender Bereiche, B. C. Teubner, Leipzig und Berlin, 1913

[2] Kaplan W., Close to convex schlicht functions, Michigan Math. J., 1952, 1, 169-185

[3] Ozaki S., On the theory of multivalent functions, Sci. Rep. Tokyo Bunrika Daig. A, 1935, 2, 167-188

[4] Umezawa T., On the theory of univalent functions, Tohoku Math. J., 1955, 7, 212-228

[5] Umezawa T., Multivalently close-to-convex functions, Proc. Amer. Math. Soc., 1957, 8, 869-874

[6] Lewandowski Z., Sur l'identité de certaines classes de fonctions univalentes, I, Ann. Univ. Mariae Curie-Skłodowska Sect. A, 1958, 12, 131-146

[7] Lewandowski Z., Sur l’identité de certaines classes de fonctions univalentes, II, Ann. Univ. Mariae Curie-Skłodowska Sect. A, 1960, 14, 19-46

[8] Nunokawa M., Sokót J., On some lenght problems for close-to-convex functions, Studia Scient. Math. Hungarica, 2018, 55(3), 293-304

[9] Bazilevič I. E., On the case of integrability in quadratures of Loewner-Kufariev equation, Mat. Sb., 1955, 37, 47-476 (in Russian)

[10] Thomas D. K., On Bazilevič functions, Trans. Amer. Math. Soc., 1968, 132, 353-361

[11] Arif M., Raza M., Noor K. I., Malik S. N., On strongly Bazilevič functions associated with generalized Robertson functions, J. Math. Comput. Model., 2011, 54(5-6), 1608-1612

[12] Haq W. U., Arif M., Khan A., Arc length inequality for a certain class of analytic functions related to conic regions, J. Complex Analysis, 2013 (2013), Article ID 407596

[13] Noor K. I., Al-Bany S. A., On Bazilevič functions, Inter. J. Math. Math. Sci., 1987, 10(1), 79-88

[14] Noor K. I., On generalized Bazilevic functions related with conic regions, Abstract Appl. Analysis, 2012(2012), Article ID 345261

[15] Noor K. I., Yousaf K., On uniformly Bazilevič and related functions, J. Appl. Math., 2012(2012), Article ID 982321

[16] Thomas D. K., A note on starlike functions, J. London Math. Soc., 1968, 43, 703-706

[17] Thomas D. K., On starlike and close-to-convex univalent functions, J. London Math. Soc., 1967, 42, 427-435

[18] Nunokawa M., On Bazilevič and convex functions, Trans. Amer. Math. Soc., 1969, 143, 337-341

[19] Nunokawa M., Sokót J., On some lenght problems for analytic functions, Osaka J. Math., 2014, 51, 695-707

[20] Nunokawa M., Sokót J., On some lenght problems for univalent functions, Math. Meth. Appl. Sci., 2016, 39(7), 1662-1666

[21] Pommerenke Ch., Über nahezu konvexe analitische Functionen, Arch. Math., 1965, 16, 344-347

[22] Nunokawa M., On Bazilevič functions of bounded boundary rotation, J. Math. Soc. Japan, 1972, 24(2), 275-278 